\title{
A comparative study of cyclophosphamide, thalidomide and dexamethasone (CTD) versus bortezomib and dexamethasone (BDex) in light-chain amyloidosis
}

Baojian Liu

Xijing Hospital https://orcid.org/0000-0002-3990-8652

Yan Wang

Xijing Hospital

Xiaoxuan Ning

Xijing Hospital

Ming Bai

Xijing Hospital

Di Wang

Xijing Hospital

Jin Zhao

Xijing Hospital

Meilan Zhou

Xijing Hospital

Shiren Sun ( $\nabla$ sunshiren@medmail.com.cn )

\section{Research}

Keywords: light chain amyloidosis, bortezomib, cyclophosphamide, thalidomide, dexamethasone

Posted Date: June 17th, 2020

DOl: https://doi.org/10.21203/rs.3.rs-35904/v1

License: (c) (i) This work is licensed under a Creative Commons Attribution 4.0 International License. Read Full License

Version of Record: A version of this preprint was published at Current Problems in Cancer on April 1st, 2021. See the published version at https://doi.org/10.1016/j.currproblcancer.2020.100669. 


\section{Abstract}

Background: Cyclophosphamide, thalidomide and dexamethasone (CTD) or bortezomib and dexamethasone (BDex) show substantial efficacy in patients with amyloid light-chain ( $A L)$ amyloidosis, especially in Chinese patients. Currently, both regimens are recommended as primary treatment options for $\mathrm{AL}$ amyloidosis, but no comparative study has been reported.

Results: We retrospectively evaluated the outcomes of $81 \mathrm{AL}$ patients who received CTD $(n=42)$ or BDex $(n=39)$ and used Mayo stage 2012 to match 26 pairs of patients. In the whole cohort, the overall hematologic responses were $86 \%$ vs $91 \%$ in the CTD and BDex groups, including a complete response of $56 \%$ vs $71 \%$ based on an intention-to-treat (ITT) analysis. Oneand two-year overall survival (OS) was $90.2 \%$ and $81.7 \%$ with CTD, and $87.6 \%$ and $82.7 \%$ with BDex. After matching, BDex regimen induced a significantly deeper and more rapid hematologic response over CTD, but no statistically significant difference in OS (ITT analysis, $\mathrm{P}=0.24 ; 6$-month landmark analysis, $\mathrm{P}=0.48$ ). Cardiac response rates were similar, while there was a trend for higher renal responses in patients treated with BDex (68\% vs 44\%, $P=0.09)$. Additionally, BDex was associated with significantly improved survival in patients with advanced disease (Mayo stage III or worse) $(P=0.009)$. Patients treated with BDex reported more episodes of severe hematologic toxicity and diarrhea.

Conclusions: CTD and BDex are effective treatments for Chinese patients with AL amyloidosis, but BDex regimen appears superior to CTD in achieving a more rapid and deeper clonal response, and in improving OS in patients with advanced disease.

\section{Background}

Amyloid light-chain (AL) amyloidosis is characterized by the deposition of misfolded immunoglobulin light chains derived from clonal plasma cells, which leads to multi-organ dysfunction. ${ }^{1}$ Between 2007 and 2015, the prevalence of AL amyloidosis in United States increased from 15.5 to 40.5 per million person-years, with an annual percentage change of $12 \%{ }^{2}$ Although rare, the prognosis of AL patients remains poor with an estimated 4-year overall survival (OS) of $50 \%$ even when treated with novel agents. ${ }^{3}$ The most frequently affected organs include kidney and heart, of which the severity of cardiac dysfunction determines survival. ${ }^{4}$ Approximately $70 \%$ of patients have renal involvement at diagnosis and one third of patients progress to dialysis after a median follow-up of 50 months. ${ }^{5}$ These have serious consequences for individuals both in terms of the economic burden ${ }^{6}$ and quality of life $\mathrm{e}^{7}$. AL amyloidosis is the most frequently diagnoses type of systemic amyloid disease in China, and the incidence is increasing, possibly due to a combination of improved diagnostic techniques and an aging population. ${ }^{8}$ Therefore, developing an optimal therapeutic schedule for Chinese AL patients to reduce mortality and improve quality of life is of great importance.

At present, the therapeutic target of $\mathrm{AL}$ amyloidosis treatment is destruction of monoclonal plasma cells to eliminate the production of the toxic amyloidogenic light chains. ${ }^{3}$ Therefore, most of the effective regimens for the treatment of fragile amyloid patients are adapted from treatments for patients with multiple myeloma (MM). ${ }^{4}$ Bortezomib-alkylator-steroid triplet combinations are the most commonly used first-line therapies for treating patients with AL who are not eligible for transplant. But the two-drug combination of bortezomib + dexamethasone (BDex) also achieves deep hematologic responses, especially among Asians, and remain widely used. ${ }^{9-13}$ A European study showed that the addition of cyclophosphamide to the BDex regimen had no significant impact on efficacy. ${ }^{14}$ Considering the high activity of bortezomib on plasma cells and the low tumor burden of AL amyloidosis, BDex regimen was recommended for treating newly diagnosed patients not eligible for transplant in Mayo Stratification of Myeloma and Risk-Adapted Therapy (mSMART) Consensus Statement published in 2015. ${ }^{15}$ Studies conducted in the United Kingdom showed high clonal response rates using cyclophosphamide, thalidomide and dexamethasone (CTD), and this treatment has been used for

first-line therapy for AL amyloidosis over the last decade in the United Kingdom. ${ }^{15-18}$ Although some studies have reported that thalidomide-related toxic effects were significant, two prospective studies (including one randomized controlled trial)

Page 2/14 
suggested that CTD-related toxicities were less frequent than those following use of the single-agent. ${ }^{18,19} \mathrm{~A}$ retrospective study from our own center also showed that CTD was an effective and safe option for treating AL patients with renal involvement. ${ }^{20} \mathrm{~A}$ recently published network meta-analysis ${ }^{21}$ concluded that CTD could induce the highest rate of renal response and BDex was associated with highest cardiac response rate. There has been a retrospective comparison of cyclophosphamide, bortezomib, and dexamethasone versus CTD, ${ }^{16}$ but no direct comparison of BDex and CTD has been reported.

In our center in Shaanxi Province, China we recommend BDex or CTD as first-line therapy for patients with AL amyloidosis who are not eligible for transplantation. We conducted a retrospective study to evaluate the efficacy and safety of these treatment regimens in Chinese patients with $\mathrm{AL}$ amyloidosis.

\section{Results}

A total of 81 patients were included. The median age of all patients was 56 years and $51 \%$ were male. The majority of patients (97.5\%) had renal involvement with a median estimated glomerular filtration rate (eGFR) of $78.1 \mathrm{~mL} / \mathrm{min} / 1.73 \mathrm{~m} 2$ and a median 24-h urinary protein of $3.36 \mathrm{~g}$. Cardiac involvement was present in 61 (75.3\%) patients, and Mayo stage distribution was $39.5 \%, 25.9 \%, 25.9 \%$ and $8.6 \%$ for stages I, II, III, and IV, respectively. Of the 81 patients included, 42 were treated with CTD and 39 with BDex (Table 1). The median number of cycles received in each group was similar (8.0 for CTD group and 6.0 for BDex group, $P=0.235$ ). Most of the parameters were comparable between the two treatment groups but variables associated with cardiac involvement, N-terminal pro-B-type natriuretic peptide (NT-proBNP), troponin T (TnT), and intraventricular septum (IVS), and clonal depth measured as the difference between involved and uninvolved free light chain (dFLC) were significantly higher in the BDex group (Table 1). All of the above variables are known to be independent prognostic factors of OS. Therefore, we matched patients by Mayo stage 2012, which includes NT-proBNP, TnT, and dFLC.

There were 26 patients in each treatment group in the matched cohort. After matching, there were no significant differences in patients' characteristics in terms of any clinical or biochemical characteristic, including NT-proBNP, TnT, IVS, dFLC, and the distribution of organ involvement (Table 2). There were also no differences in the median follow-up time (22.5m for the CTD group vs $15.0 \mathrm{~m}$ for the BDex group, $P=0.210$ ) and the number of cycles received (5.0 vs 7.5 respectively, $P=0.761$ ) between two groups.

\section{$\underline{\text { Hematologic and organ response }}$}

In the ITT analysis, the overall hematologic response (OHR) rates in the CTD and BDex group were $86 \%$ vs $91 \%(P=0.77)$, including a complete response (CR) rate in $56 \%$ vs $71 \%(P=0.19)$ of the primary cohort (Table 3$)$. Given that rapid clonal control is strongly associated with better OS and preservation of organ function, ${ }^{22} 3$-month and 6-month hematologic responses were also evaluated among the evaluable patients. There were no differences in OHR between treatment groups at either 3 or 6 months. However, there was a significant improvement in the rate of CR in patients treated with BDex (47\% vs $19 \%$ at 3 months, $P=0.02 ; 69 \%$ vs $38 \%$ at 6 months, $P=0.02$ ).

In the ITT analysis of the matched sub-cohort, the OHR rates was $75 \%$ in the CTD group vs $96 \%$ in the BDex group ( $P=$ 0.13). The rate of $\mathrm{CR}$ was $45 \%$ and $74 \%$, respectively $(P=0.053)$ (Table 3$)$. A significantly higher $\mathrm{CR}$ rate was observed at 6 months in the BDex group compared to the CTD group (75\% vs 27\%, $P=0.007)$, with a trend for a higher CR rate at 3 months (44\% vs $16 \%, P=0.053)$. The median time to initial response was 2.0 months in both treatment groups $(P=0.209)$ and median time to best response was 8.0 in the CTD group vs 2.25 months in the BDex group $(P=0.014)$.

During the follow-up period, a total of 4 (4.9\%) patients progressed to dialysis ( 3 in the CTD group and 1 in the BDex group), although two of them achieved a hematologic CR. Renal and cardiac responses were recorded in $63 \%$ and $40 \%$ of patients treated with CTD, whereas for BDex-treated patients the response rates were $72 \%$ and $46 \%$, respectively $(P>0.05$ for all comparisons). Results in matched sub-cohort showed an advantageous tendency toward preserving renal function in the

Page 3/14 
BDex group (68\% vs $44 \%$ in the CTD group, $P=0.09)$, with similar cardiac response rates in both groups $(27 \%$ vs $50 \%$ for CTD and BDex, respectively, $P=0.43$ ).

\section{Survival}

The median follow-up time in the whole cohort was 25.8 months for the CTD group and 11.0 months for the BDex group. Among the 81 patients, twelve patients died ( 8 in the CTD group and 4 in the BDex group) during the follow-up period, of whom ten were Mayo stage III or worse at diagnosis. Median OS was not reached in either group and there was no significant difference in OS $(P=0.763$, Figure $1 \mathrm{~A})$. One- and two-year OS was $90.2 \%, 81.7 \%$, respectively in patients who received the CTD regimen, vs $87.6 \%$ and $82.7 \%$ in patients who received the BDex regimen. In the 6-month landmark analysis that excluded patients whose follow-up time were less than 6 months, there were 37 patients in the CTD group and 27 in the BDex group and no significant differences in OS were observed ( $P=0.780$, Figure 1B). For the matched cohort, median OS was not reached in either group based on the ITT analysis or the 6-month landmark analysis, and no correlation between longer survival and treatment was observed $(P=0.238$ for ITT analysis and $P=0.476$ for 6 -month landmark analysis, Figure $2 \mathrm{~A}$ and $2 \mathrm{~B}$ ).

In the ITT analysis we next tested the effect of treatment regimen on patients with Mayo stage $\leq$ II or $>$ II to find out if the two regimens had different effects in treating patients with different risk levels. The results indicated that subjects with advanced (Mayo stage > II) AL amyloidosis who received BDex survived significantly longer than those who received CTD (median survival not reached vs. 11.5 months, $P=0.009$, Figure 3, c vs d). While OS was similar following BDex or CTD in patients with Mayo stage $\leq \|(P=0.318$, Figure 3 , a vs b), although the small sample size limits interpretation.

Univariate and multivariate analyses with Cox regression were used to predict factors closely associated with survival. The variables predicting OS by univariate analysis in the whole cohort were alkaline phosphatase (ALP) level, eGFR, Mayo stage 2012, IVS, and dialysis. After adjustment for these factors in the multivariate analysis, the BDex regimen showed a protective effect on survival (hazard ratio $[\mathrm{HR}]=0.19,95 \% \mathrm{Cl}, 0.05-0.67 ; P=0.01$ ) compared to CTD regimen in treating $\mathrm{AL}$ amyloidosis (Table 4). In addition, Mayo stage III $(\mathrm{HR}=25.6,95 \% \mathrm{Cl}, 2.7-241.9 ; P=0.05)$ and Mayo stage IV $(\mathrm{HR}=23.3$, $95 \% \mathrm{Cl}, 1.1-498.8 ; P=0.044)$ were independently associated with poor OS (Table 4).

\section{Toxicity}

Overall, $76.9 \%$ of patients treated with BDex and $64.3 \%$ of patients treated with CTD reported at least one adverse event during the study $(P=0.213)$ (Table 5$)$. There were more reports of any and grade $\geq 3$ hematologic symptoms (anemia, thrombocytopenia or neutropenia) in patients treated with BDex than in those treated with CTD. Among non-hematologic toxicities, reports of diarrhea and herpes zoster were more common in the BDex than the CTD group (30.8\% vs $2.4 \%$, respectively for diarrhea and $23.1 \%$ vs $4.8 \%$ for herpes zoster). In the CTD group, fluid retention (16.7\%) and fatigue (14.3\%) were the most common non-hematologic toxicities. Few patients reported peripheral neuropathy (12.8\% in the BDex group and $9.5 \%$ in the CTD group) and no grade $\geq 3$ peripheral neuropathy was observed.

Grade $\geq 3$ adverse events were reported significantly more frequently by patients in the BDex group than the CTD group (33.3\% vs $14.3 \%, P=0.043$ ) (Table 5). Diarrhea (10.3\%) and hematologic toxicities were the most commonly reported toxicities of grade $\geq 3$ severity in the BDex group, and an acute kidney injury event occurred in one patient due to severe diarrhea. In the CTD group, one patient reported severe gastrointestinal bleeding that required admission to hospital for blood transfusion.

\section{Discussion}

To our knowledge, this is the first comparison of clinical outcomes and tolerability of BDex versus CTD in patients with AL amyloidosis. In this retrospective study, we observed encouraging rates of OHR, CR and organ response in AL patients 
treated with CTD or BDex. After matching by Mayo stage 2012, the BDex regimen appeared to achieve a more rapid and deeper clonal response compared to CTD. But no significant improvement in OS was observed in BDex group based on ITT analysis or 6-month landmark analysis. Of note however, BDex regimen did significantly improve OS in the group of patients who were Mayo stage > II. Treatment-related toxicity was manageable under both regimens, but severe hematologic toxicity and diarrhea should be managed carefully in patients treated with BDex. Our study shows that in Chinese patients, BDex is a highly active and generally well-tolerated therapy, and superior to CTD in achieving rapid and deep hematologic responses and improving survival of patients with advanced disease.

The treatment of $A L$ amyloidosis is largely derived from the experience of treating MM. However, $A L$ amyloidosis is a plasma cell dyscrasia with low tumor burden and shows exceptional sensitivity to bortezomib. ${ }^{23}$ Therefore, addition of cyclophosphamide or melphalan to the BDex regimen may be not necessary for successful treatment of AL amyloidosis. This is supported by several studies that have confirmed the activity of BDex for treating newly diagnosed and relapsed AL patients. ${ }^{9-13,24,25}$ Currently, there is no standard treatment for AL amyloidosis, although bortezomib-based chemotherapy regimens are the preferred choice in many western countries. Differences in disease patterns are present between East and West in patients with AL amyloidosis, ${ }^{8}$ and the optimal treatment schedule for Chinese patients needs further exploration. CTD regimen as alternative frontline treatment for $A L$ amyloidosis has been reported in many studies, ${ }^{15,17,26}$ and its effect in our center has also been validated. ${ }^{20}$

We observed very high clinical response rates, with an OHR of at least $86 \%$ and CR rates of at least $56 \%$ following either treatment, which is higher than responses previously reported following other chemotherapies. ${ }^{27-29}$ There were some differences in the demographic and clinical features of patients in our center compared with reports from western countries. ${ }^{28,30}$ First, patients in our center tended to be younger than those reported in studies conducted in western countries (mean 56 years vs 63 years), but similar to another Chinese center (56 years). ${ }^{31}$ Second, there was a higher proportion of patients with renal involvement in our center (97.5\% vs $61 \%)$, reflecting that recruitment of patients from the department of nephrology. Third, a low Mayo stage 2012 ( $\leq$ Mayo stage III) was more common in patients at our center (65\% vs 43\%). Fourth, patients with AL amyloidosis in our center had a good renal function (eGFR, 78 vs

$64 \mathrm{~mL} / \mathrm{min} / 1.73 \mathrm{~m}^{2}$ ). These differences might partially explain why the patients in our center had excellent hematologic and organ response rates to treatment.

The characteristics of patients at baseline were not balanced between treatment groups, with a higher number of patients with advanced disease particularly cardiac involvement in the BDex group. This could be due to the selection of more advanced patients to receive bortezomib, which is more costly than CTD and so potentially reserved for advanced disease. In the matched cohort, BDex showed a trend for a higher rate of CR at 3 months and a clear difference at 6 months compared to CTD, indicating a rapid and deep hematologic response to BDex therapy. This was in accordance with a significantly shorter time to best hematologic response in patients treated with BDex. Nevertheless, the deeper hematologic responses was not associated with a significant improvement in organ function, although some patients did not complete the full cycle and follow-up time was relatively short, which may account for this phenomenon.

The median OS was not reached in either group due to the relative short follow-up time and there was no significant difference between patients treated with CTD and BDex among the matched cohort or 6-month landmark analysis. The significantly improved survival in the sub-group of patients with advanced disease (Mayo stage 2012 III or IV) who received BDex raises the hypothesis that the choice of chemotherapy regimen should be based on risk stratification. Based on our results, either CTD or BDex is suitable for patients with Mayo stage I or II, whereas for patients with Mayo stage III or IV, BDex may be the optimal choice. This hypothesis warrants further verification in larger populations. In addition, because BDex showed a potential protective effect in achieving hematologic and organ responses compared with CTD, we incorporated it into the multivariate analysis although it was not significant at the univariate analysis. After adjustment 
BDex was associated with improved survival, suggesting that BDex might be superior to CTD regimen in improving OS in selected AL patients.

Some studies have reported that patients with $\mathrm{AL}$ amyloidosis tolerate high dose of thalidomide poorly. ${ }^{32,33}$ Therefore the CTD regimen used in our center refers to the attenuated regimen, ${ }^{18}$ which has similar efficacy but with less toxicity compared with the standard CTD regimen. Although the total incidence of adverse events exceeded $60 \%$, most were of mild to moderate severity and were tolerated by patients. We used once-weekly subcutaneous bortezomib combined with dexamethasone as the standard therapy at our center based on the recommendations of the British Committee for Standards in Hematology. ${ }^{34}$ Almost one-quarter of patients treated with BDex developed herpes zoster, suggesting that anti-viral prophylaxis is essential for AL patients treated with BDex. While peripheral neuropathy is a major dose-limiting side-effect of bortezomib treatment, peripheral neuropathy occurred in only $12.8 \%$ of patients in our study, which is lower than most previous estimates reported in AL patients. ${ }^{35}$

Although the results are encouraging, there were several study limitations that prevent us to draw meaningful conclusions. First, the study was retrospective and the choice of treatment subject to selection bias and we constructed a matched cohort to reduce the risk of bias between the treatment groups. Extensive efforts were made to follow up all patients, but a fraction of patients did not attend outpatient clinics prior to the end of the observation period, resulting in loss of information. We excluded patients who received less than one cycle of treatment and those who were lost to follow-up, which could have led to overestimation of response rates and survival. Because some patients were too heavy to tolerate treatment and this fraction of patients were not included. The follow-up period was relatively short and the number of enrolled subjects was small. Therefore, the hard endpoint (OS) could not be evaluated and the long-term prognosis of these patients remains uncertain. Follow-up of this cohort is ingoing in expectation of providing long-term outcome data. Finally, differentiation between treatment-related and disease-related adverse events in this multi-organ disease was challenging. Events such as fluid retention, syncope due to hypotension, and peripheral neuropathy are common presentations of $\mathrm{AL}$ amyloidosis. Therefore, not all of the reported adverse events can be assumed to be treatment-related.

\section{Conclusions}

This comparative study showed that both CTD and BDex are effective and generally well-tolerated regimens offering high hematologic and organ response rates in patients with $\mathrm{AL}$ amyloidosis in China. BDex was associated with more toxicity than CTD, but appeared to be a better option, achieving a rapid and deep clonal response, and improving survival in patients with advanced disease. These potential advantages and impacts on long-term prognosis need to be verified in further studies.

\section{Methods}

This retrospective cohort included 81 consecutive patients with biopsy-confirmed AL amyloidosis, all of whom were treated with BDex or CTD at the Department of Nephrology Xijing Hospital, Fourth Military Medical University, Xi'an, Shaanxi, China, between January 2015 and November 2019. The choice of treatment was based on physicians' recommendations and patients' discretion. Amyloidosis was confirmed by the presence of Congo red positive fibril deposition and non-branding fibrils 8-10 nm in diameter. Immunofluorescence and mass spectroscopy were used to determine the AL subtype. Patients who were treated with less than one chemotherapy cycle, who were diagnosed with MM-associated AL amyloidosis, or were lost to follow-up during the study period, were excluded. Evaluation of organ involvement and assessment of organ and hematologic responses were based on published criteria for the evaluation of AL amyloidosis. ${ }^{36-38}$ In particular, the recently established renal response criteria were used to evaluate the outcome of treatment on renal status. For the intention-to-treat (ITT) analysis, the best response achieved before relapse was recorded regardless of whether there was a 
change in treatment or not. Patients who died within 2 months before a response could be assessed were deemed nonresponders.

OS was calculated from start of treatment to death or the last follow-up visit. Given the high rate of early death in $\mathrm{AL}$ amyloidosis, we performed a 6-month landmark analysis to assess the effect of each regimen on those patients whose survival time was long enough to benefit from a full course of therapy. Toxicity was assessed and graded according to the Common Terminology Criteria for Adverse Events version 4.0..

The BDex schedule consisted of subcutaneous bortezomib at a dose of $1.3 \mathrm{mg} / \mathrm{m}^{2}$ on days $1,8,15,22$ and oral dexamethasone $20 \mathrm{mg}$ on days $1-2,8-9,15-16,22-23$, every 35 days. The CTD regimen was delivered as previously described in our center. ${ }^{20}$ The dose of both regimens could be modified at the discretion of the treating clinicians.

Background prognostic variables in the two treatment exposure groups were not balanced. Therefore, we created a subcohort of patients in each treatment group who were matched according to Mayo stage 2012, which takes into account key prognostic factors and thus eliminated this risk of bias from the analysis. When more than one case was matched, we chose the one whose value was closest to that in another group.

\section{Statistical analysis}

Continuous variables were presented as mean (standard deviation) or median (interquartile range; IQR), and categorical data were presented as frequencies. Differences between treatment exposures were compared with the student's $t$ test or the Mann-Whitney U-test for quantitative variables, and with the $X^{2}$-test or Fisher's exact test for qualitative variables.

Survival curves were plotted using the Kaplan-Meier method and compared with the log-rank test. Cox proportional-hazards regression models were fitted to identify factors predicting survival in the multivariate analysis. A two-sided $P$ value $<0.05$ was considered significant. Analyses were performed using SPSS version 24.0 (IBM, Armonk, NY).

\section{Declarations}

Ethics approval and consent to participate: The study was approved by our institution's ethics committee council (Xijing Hospital). Informed consent for study participation was obtained from individuals in accordance with the Declaration of Helsinki.

Consent for publication: Not applicable

Availability of data and materials: The datasets used and analysed during the current study are available from the corresponding author on reasonable request.

Competing interests: The authors declare that they have no competing interests.

Funding: This work was supported by the National Natural Science Foundation of China grants 81600562 (Y.W.)

Authors' contributions: S.S., B.L., Y.W. designed the study; B.L., Y.W. wrote the manuscript; X.N., M.B., D.W. treated patients, collected and analyzed data; M.B., J.Z. performed statistical analysis; M.Z., J.Z. collected and analyzed data; D.W., X.N. performed histology; S.S. treated patients and critically revised the manuscript.

Acknowledgments: Not applicable.

We affirm that the results presented in this paper have not been published previously in whole or part, except in abstract form.

\section{References}


1. Merlini G, Bellotti V. Molecular mechanisms of amyloidosis. N Engl J Med. 2003;349(6):583-96.

2. Quock TP, Yan T, Chang E, Guthrie S, Broder MS. Epidemiology of AL amyloidosis: a real-world study using US claims data. Blood Adv. 2018;2(10):1046-53.

3. Wechalekar AD, Gillmore JD, Hawkins PN. Systemic amyloidosis. Lancet. 2016;387(10038):2641-54.

4. Merlini G, Dispenzieri A, Sanchorawala V, et al. Systemic immunoglobulin light chain amyloidosis. Nat Rev Dis Primers. 2018;4(1):38.

5. Palladini G, Hegenbart U, Milani P, et al. A staging system for renal outcome and early markers of renal response to chemotherapy in AL amyloidosis. Blood. 2014;124(15):2325-32.

6. Quock TP, Yan T, Tieu R, D'Souza A, Broder MS. Untangling the clinical and economic burden of hospitalization for cardiac amyloidosis in the United States. Clinicoecon Outcomes Res. 2019;11:431-9.

7. Warsame R, Kumar SK, Gertz MA, et al. Hematology patient reported symptom screen to assess quality of life for AL amyloidosis. Am J Hematol. 2017;92(5):435-40.

8. Huang XH, Liu ZH. The Clinical Presentation and Management of Systemic Light-Chain Amyloidosis in China. Kidney Dis (Basel). 2016;2(1):1-9.

9. Katoh N, Ueno A, Yoshida T, et al. Bortezomib-dexamethasone versus high-dose melphalan for Japanese patients with systemic light-chain (AL) amyloidosis: a retrospective single-center study. Int J Hematol. 2017;105(3):341-8.

10. Huang X, Wang Q, Chen W, Ren G, Liu Z. Bortezomib with dexamethasone as first-line treatment for AL amyloidosis with renal involvement. Amyloid. 2016;23(1):51-7.

11. Lamm W, Willenbacher W, Lang A, et al. Efficacy of the combination of bortezomib and dexamethasone in systemic AL amyloidosis. Ann Hematol. 2011;90(2):201-6.

12. Kastritis $E$, Roussou M, Gavriatopoulou M, et al. Long-term outcomes of primary systemic light chain ( $A L$ ) amyloidosis in patients treated upfront with bortezomib or lenalidomide and the importance of risk adapted strategies. Am J Hematol. 2015;90(4):E60-5.

13. Huang B, Li J, Xu X, Zheng D, Zhou Z, Liu J. Successful treatment of renal light chain (AL) amyloidosis with bortezomib and dexamethasone (VD). Pathol Biol (Paris). 2015;63(1):17-20.

14. Kastritis E, Gavriatopoulou M, Roussou M, et al. Addition of cyclophosphamide and higher doses of dexamethasone do not improve outcomes of patients with AL amyloidosis treated with bortezomib. Blood Cancer J. 2017;7(6):e570.

15. Dispenzieri A, Buadi F, Kumar SK, et al. Treatment of Immunoglobulin Light Chain Amyloidosis: Mayo Stratification of Myeloma and Risk-Adapted Therapy (mSMART) Consensus Statement. Mayo Clin Proc. 2015;90(8):1054-1081.

16. Venner CP, Gillmore JD, Sachchithanantham S, et al. A matched comparison of cyclophosphamide, bortezomib and dexamethasone (CVD) versus risk-adapted cyclophosphamide, thalidomide and dexamethasone (CTD) in AL amyloidosis. Leukemia. 2014;28(12):2304-10.

17. Rysava R. AL amyloidosis: advances in diagnostics and treatment. Nephrol Dial Transplant. 2019;34(9):1460-6.

18. Wechalekar AD, Goodman HJ, Lachmann HJ, Offer M, Hawkins PN, Gillmore JD. Safety and efficacy of risk-adapted cyclophosphamide, thalidomide, and dexamethasone in systemic AL amyloidosis. Blood. 2007;109(2):457-64.

19. Gillmore J, Cocks K, Gibbs SDJ, et al. Cyclophosphamide, thalidomide and dexamethasone (CTD) versus melphalan plus dexamethasone (MD) for newly-diagnosed systemic AL amyloidosis - results from the UK amyloidosis treatment trial. Blood. 2009;114(22):1120.

20. Liu B, Wang Y, Bai M, et al. Cyclophosphamide + Thalidomide + Dexamethasone Versus Melphalan + Dexamethasone for the Treatment of Amyloid Light-chain Amyloidosis With Kidney Involvement: A Retrospective Study in Chinese Patients. Clin Ther. 2019;41(6):1186-98.

21. Cai Y, Xu S, Li N, Li S, Xu G. Efficacy of Chemotherapies and Stem Cell Transplantation for Systemic AL Amyloidosis: A Network Meta-Analysis. Front Pharmacol. 2019;10:1601. 
22. Rezk T, Lachmann HJ, Fontana M, et al. Prolonged renal survival in light chain amyloidosis: speed and magnitude of light chain reduction is the crucial factor. Kidney Int. 2017;92(6):1476-83.

23. Oliva L, Orfanelli $U$, Resnati $M$, et al. The amyloidogenic light chain is a stressor that sensitizes plasma cells to proteasome inhibitor toxicity. Blood. 2017;129(15):2132-42.

24. Kastritis E, Anagnostopoulos A, Roussou M, et al. Treatment of light chain (AL) amyloidosis with the combination of bortezomib and dexamethasone. Haematologica. 2007;92(10):1351-8.

25. Kastritis E, Wechalekar AD, Dimopoulos MA, et al. Bortezomib with or without dexamethasone in primary systemic (light chain) amyloidosis. J Clin Oncol. 2010;28(6):1031-7.

26. Merlini G, Wechalekar AD, Palladini G. Systemic light chain amyloidosis: an update for treating physicians. Blood. 2013;121(26):5124-30.

27. Palladini G, Sachchithanantham S, Milani P, et al. A European collaborative study of cyclophosphamide, bortezomib, and dexamethasone in upfront treatment of systemic AL amyloidosis. Blood. 2015;126(5):612-5.

28. Manwani R, Cohen O, Sharpley F, et al. A prospective observational study of 915 patients with systemic AL amyloidosis treated with upfront bortezomib. Blood. 2019;134(25):2271-80.

29. Palladini G, Milani P, Foli A, et al. Melphalan and dexamethasone with or without bortezomib in newly diagnosed AL amyloidosis: a matched case-control study on 174 patients. Leukemia. 2014;28(12):2311-6.

30. Muchtar E, Gertz MA, Kumar SK, et al. Improved outcomes for newly diagnosed AL amyloidosis between 2000 and 2014: cracking the glass ceiling of early death. Blood. 2017;129(15):2111-9.

31. Huang X, Wang Q, Jiang S, Chen W, Zeng C, Liu Z. The clinical features and outcomes of systemic AL amyloidosis: a cohort of 231 Chinese patients. Clin Kidney J. 2015;8(1):120-6.

32. Dispenzieri A, Lacy MQ, Rajkumar SV, et al. Poor tolerance to high doses of thalidomide in patients with primary systemic amyloidosis. Amyloid. 2003;10(4):257-61.

33. Palladini G, Perfetti V, Perlini S, et al. The combination of thalidomide and intermediate-dose dexamethasone is an effective but toxic treatment for patients with primary amyloidosis (AL). Blood. 2005;105(7):2949-51.

34. Wechalekar AD, Gillmore JD, Bird J, et al. Guidelines on the management of AL amyloidosis. Br J Haematol. 2015;168(2):186-206.

35. Liu B, Bai M, Wang Y, et al. The efficacy and safety of bortezomib-based chemotherapy for immunoglobulin light chain amyloidosis: A systematic review and meta-analysis. Eur J Intern Med. 2019;69:32-41.

36. Gillmore JD, Wechalekar A, Bird J, et al. Guidelines on the diagnosis and investigation of AL amyloidosis. Br J Haematol. 2015;168(2):207-18.

37. Comenzo RL, Reece D, Palladini G, et al. Consensus guidelines for the conduct and reporting of clinical trials in systemic light-chain amyloidosis. Leukemia. 2012;26(11):2317-25.

38. Palladini G, Dispenzieri A, Gertz MA, et al. New criteria for response to treatment in immunoglobulin light chain amyloidosis based on free light chain measurement and cardiac biomarkers: impact on survival outcomes. $\mathrm{J}$ Clin Oncol. 2012;30(36):4541-9.

\section{Tables}

Table 1. Characteristics of all patients at baseline. 


\begin{tabular}{|c|c|c|c|}
\hline Variables & CTD group $(n=42)$ & BDex group (n=39) & $\mathrm{P}$ \\
\hline Age (y) mean \pm SD & $57 \pm 9$ & $56 \pm 10$ & 0.75 \\
\hline Male n (\%) & $18 \overline{(43)}$ & $23(59)$ & 0.15 \\
\hline Time to diagnosis (mo.) median (IQR) & $8(5-17)$ & $6(3-20)$ & 0.66 \\
\hline $\mathrm{SBP}<100 \mathrm{mmHg} \mathrm{n}(\%)$ & $13(31)$ & $15(39)$ & 0.48 \\
\hline Alb $(g / d L)$ mean \pm SD & $26 \pm 6$ & $28 \pm 8$ & 0.26 \\
\hline ALP (IU/L) median (IQR) & $72(53-92)$ & $74(59-104)$ & 0.38 \\
\hline$\lambda$ Subtype $n(\%)$ & $32(76)$ & $35(90)$ & 0.11 \\
\hline dFLC (mg/L) median (IQR) & $44(19-99) \mathrm{n}=37$ & $102(50-157)$ & 0.002 \\
\hline NT-proBÑP' (ng/L) median (IQR) & $537(158-1666)$ & $1343(738-4491)$ & 0.004 \\
\hline $\operatorname{TnT}(\mu \mathrm{g} / \mathrm{L})$ median (IQR) & $0.02(0.01-0.05) \mathrm{n}=39$ & $0.05(0.03-0.07) \mathrm{n}=38$ & 0.001 \\
\hline $\operatorname{TnI}(\mu \mathrm{g} / \mathrm{L})$ median (IQR) & $0.02(0.01-0.07)$ & $0.05(0.02-0.15)$ & 0.04 \\
\hline IVS (cm) median (IQ̀R) & $1.1(1.0-1.2)$ & $1.3(1.0-1.5)$ & 0.003 \\
\hline $\mathrm{EF}(\%)$ mean $\pm \mathrm{SD}$ & $59 \pm 4$ & $57 \pm 4$ & 0.08 \\
\hline 24-h urinary protein (g) median (IQR) & $3.0 \overline{2}(1.61-4.22)$ & $4.5 \overline{0}(1.58-7.21)$ & 0.11 \\
\hline Urinary protein $>3.5 \mathrm{~g} / 24 \mathrm{~h} \mathrm{n}(\%)$ & $18(43)$ & $22(56)$ & 0.22 \\
\hline eGFR (mL/min $\left./ 1.73 \mathrm{~m}^{2}\right)$ median (IQR) & $78(58-96)$ & $(59-97)$ & 0.55 \\
\hline Second-line treatment n (\%) & $8(19)$ & $3(8)$ & 0.14 \\
\hline Switched therapy $\mathrm{n}(\%)$ & $5(12)$ & $2(5)$ & 0.49 \\
\hline \multirow{8}{*}{$\begin{array}{l}\text { Involved organ n (\%) } \\
\text { Heart } \\
\text { Kidney } \\
\text { Liver } \\
\text { Mayo stage } 2012 \text { n (\%) }\end{array}$} & & & \multirow{8}{*}{$\begin{array}{l}0.004 \\
0.23 \\
1.0 \\
00.001\end{array}$} \\
\hline & $\begin{array}{ll}26 \\
42(102)\end{array}$ & $\begin{array}{l}35(90) \\
37(95)\end{array}$ & \\
\hline & $4(10)$ & $4(10)$ & \\
\hline & & & \\
\hline & $24(57)$ & $8(21)$ & \\
\hline & $10(24)$ & $11(28)$ & \\
\hline & $7(17)$ & $14(36)$ & \\
\hline & $1(2)$ & $6(15)$ & \\
\hline
\end{tabular}

SD, standard deviation; IQR, interquartile range; SBP, systolic blood pressure; Alb, albumin; ALP, alkaline phosphatase; dFLC, difference between involved and uninvolved free light chain; NT-proBNP, N-terminal pro-B-type natriuretic peptide; TnT, troponin T; TnI, troponin I; IVS, intraventricular septum; EF, ejection fraction; eGFR, estimated glomerular filtration rate.

Table 2. Characteristics of matched patients at baseline.

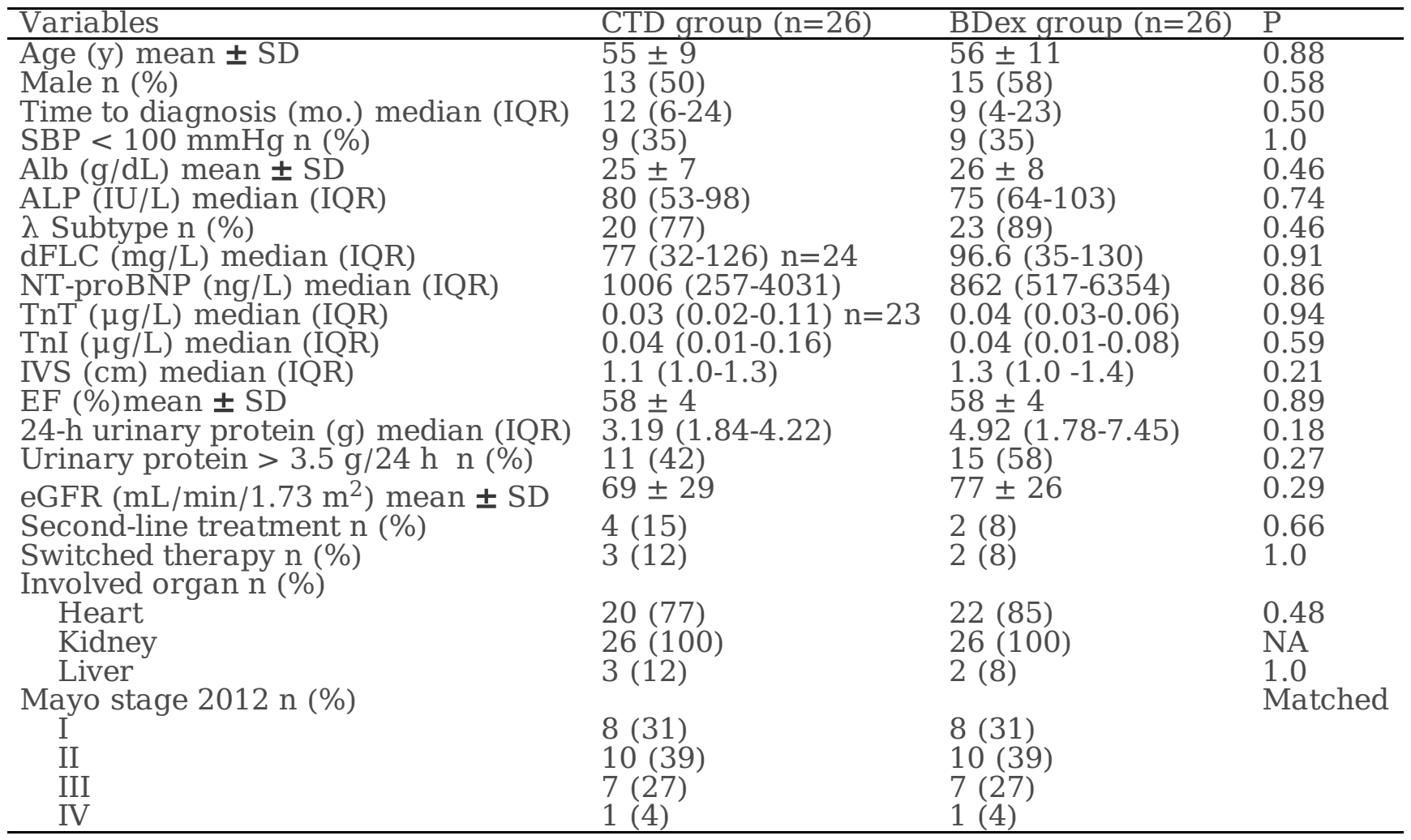


SD, standard deviation; IQR, interquartile range; SBP, systolic blood pressure; Alb, albumin; ALP, alkaline phosphatase; dFLC, difference between involved and uninvolved free light chain; NT-proBNP, N-terminal pro-B-type natriuretic peptide; TnT, troponin T; TnI, troponin I; IVS, intraventricular septum; EF, ejection fraction; eGFR, estimated glomerular filtration rate; NA, not available.

Table 3. Hematologic and organ response

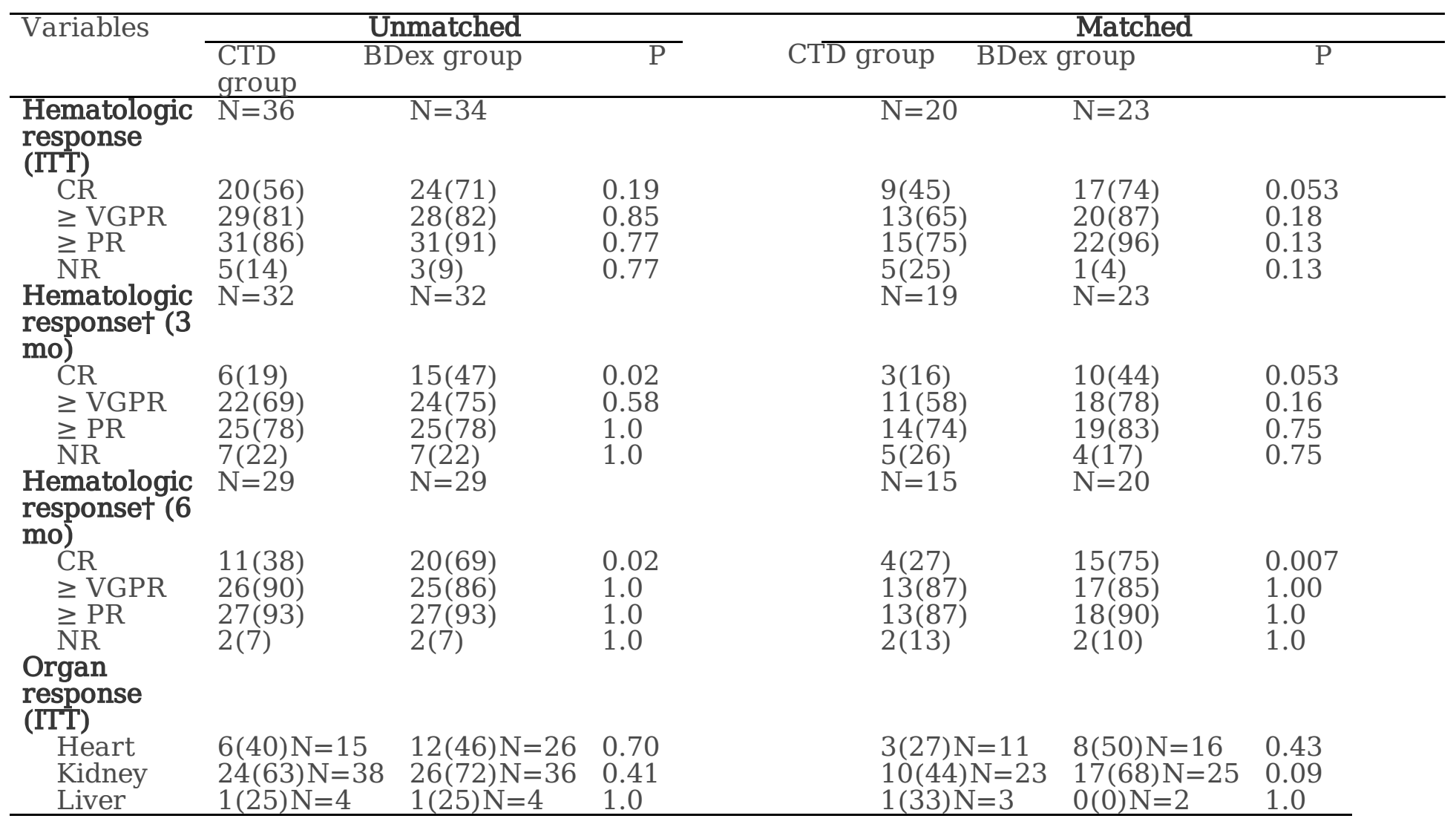

CR, complete response; VGPR, very good partial response; PR, partial response; NR, no response; ITT, intention-to-treat.

Data are given as number (\%) of patients.

$\mathrm{N}$ values represent the evaluable patients.

† Analysis was conducted after 3/6 months of therapy.

Table 4. Univariate and multivariate analysis of factors associated with overall survival 


\begin{tabular}{|c|c|c|c|c|}
\hline \multirow[t]{2}{*}{ Variables } & \multicolumn{2}{|l|}{ Univariate } & \multicolumn{2}{|l|}{ Multivariate } \\
\hline & HR (95\% CI) & $\mathrm{P}$ & HR (95\% CI) & $\mathrm{P}$ \\
\hline Age $>65(y)$ & $1.67(0.45-6.19)$ & 0.44 & & NI \\
\hline Male & $2.88(0.86-9.64)$ & 0.09 & & NI \\
\hline Alb (g/dL) & $0.96(0.88-1.04)$ & 0.31 & & NI \\
\hline $\mathrm{SBP}<100(\mathrm{mmHg})$ & $1.55(0.49-4.89)$ & 0.46 & & NI \\
\hline к subtype & $0.67(0.14-3.10)$ & 0.61 & & NI \\
\hline BDex & $0.83(0.25-2.81)$ & 0.76 & $0.19(0.05-0.67)$ & 0.01 \\
\hline ALP $>87.5(\mathrm{IU} / \mathrm{L})$ & $5.09(1.52-16.97)$ & 0.008 & NS & 0.24 \\
\hline $\mathrm{eGFR}<60\left(\mathrm{~mL} / \mathrm{min} / 1.73 \mathrm{~m}^{2}\right)$ & $3.54(1.12-11.17)$ & 0.03 & NS & 0.82 \\
\hline Mayo stage I & 1 & & 1 & \\
\hline Mayo stage II & $1.92(0.12-30.80)$ & 0.64 & $1.66(0.10-27.25)$ & 0.72 \\
\hline Mayo stage III & $24.9(3.0-204.7)$ & 0.003 & $25.6(2.7-241.9)$ & 0.05 \\
\hline Mayo stage IV & $28.1(1.5-537.7)$ & 0.03 & $23.3(1.1-498.8)$ & 0.04 \\
\hline IVS $\geq 1.2(\mathrm{~cm})$ & $16.1(2.1-125.0)$ & 0.008 & $8.1(0.98-67.6)$ & 0.052 \\
\hline Dialysis & $5.40(1.42-20.47)$ & 0.01 & NS & 0.11 \\
\hline
\end{tabular}

CI, confidence interval; HR, hazard ratio; Alb, albumin; SBP, systolic blood pressure; ALP, alkaline phosphatase; eGFR, estimated glomerular filtration rate; IVS, intraventricular septum; NI, not included; NS, not significant.

Table 5. Toxicities reported in patients with AL amyloidosis treated with BDex or CTD

\begin{tabular}{|c|c|c|c|c|c|c|}
\hline \multirow[t]{3}{*}{ Events } & \multicolumn{3}{|c|}{ All grades n(\%) } & \multicolumn{3}{|c|}{ Grade $\geq 3 n(\%)$} \\
\hline & CTD & BDex & $\bar{P}$ & CTD & BDex & $\mathrm{P}$ \\
\hline & $(n=42)$ & $(n=39)$ & & $(\mathrm{n}=42)$ & $(n=39)$ & \\
\hline Anemia & $6(14.3)$ & $13(33.3)$ & 0.04 & 0 & $2(5.1)$ & 0.23 \\
\hline Thrombocytopenia & $2(4.8)$ & $8(20.5)$ & 0.07 & 0 & $3(7.7)$ & 0.21 \\
\hline Neutropenia & $2(4.8)$ & $4(10.3)$ & 0.60 & $1(2.4)$ & $3(7.7)$ & 0.56 \\
\hline Fatigue & $6(14.3)$ & $6(15.4)$ & 0.89 & & & NA \\
\hline Infection & $2(4.8)$ & $3(7.7)$ & 0.93 & $2(4.8)$ & $2(5.1)$ & 1.00 \\
\hline Diarrhea & $1(2.4)$ & $12(30.8)$ & 0.001 & 0 & $4(10.3)$ & 0.11 \\
\hline Constipation & $3(7.1)$ & $5(12.8)$ & 0.63 & 0 & & NA \\
\hline Nausea/vomit & $2(4.8)$ & $2(5.1)$ & 1.00 & 0 & 0 & NA \\
\hline Syncope & $2(4.8)$ & $2(5.1)$ & 1.00 & $2(4.8)$ & $2(5.1)$ & 1.00 \\
\hline Tremor & $2(4.8)$ & 0 & 0.49 & 0 & 0 & NA \\
\hline Peripheral neuropathy & $4(9.5)$ & $5(12.8)$ & 0.91 & 0 & 0 & NA \\
\hline Herpes zoster & $2(4.8)$ & $9(23.1)$ & 0.02 & 0 & 0 & NA \\
\hline Rash & & $3(7.7)$ & 0.21 & 0 & 0 & NA \\
\hline Fluid retention & $7(16.7)$ & $5(12.8)$ & 0.63 & 0 & 0 & NA \\
\hline Acute kidney injury & 0 & $1(2.6)$ & 0.48 & 0 & $1(2.6)$ & 0.48 \\
\hline Ileus & 0 & $1(2.6)$ & 0.48 & 0 & 0 & NA \\
\hline Gastrointestinal bleeding & $1(2.4)$ & 0 & 1.0 & $1(2.4)$ & 0 & 1.00 \\
\hline Total & $27(64.3)$ & $30(76.9)$ & 0.21 & $6(14.3)$ & $13(33.3)$ & 0.04 \\
\hline
\end{tabular}

NA, not available.

\section{Figures}




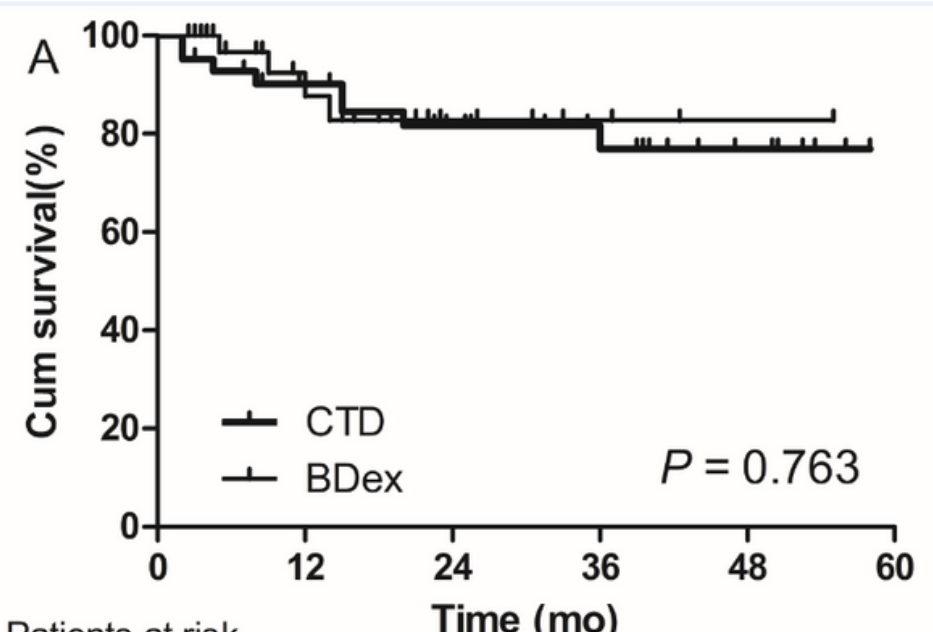

Patients at risk CTD 42

BDex 39

34

19

\section{Time (mo)}

$\begin{array}{cccc}24 & 17 & 9 & 2 \\ 8 & 4 & 2 & 1\end{array}$

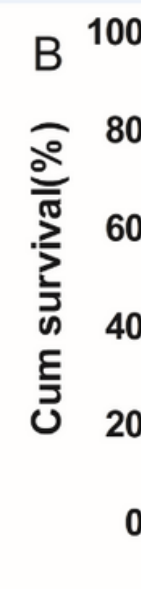

Patients at risk $\begin{array}{lllll}\text { CTD } & 37 & 34 & 24 & 17\end{array}$ $\begin{array}{llll}\text { BDex } & 27 & 19 & 8\end{array}$

\section{Figure 1}

Overall survival of patients according to treatment type based on ITT analysis (A) and 6-month landmark analysis (B) before matching.

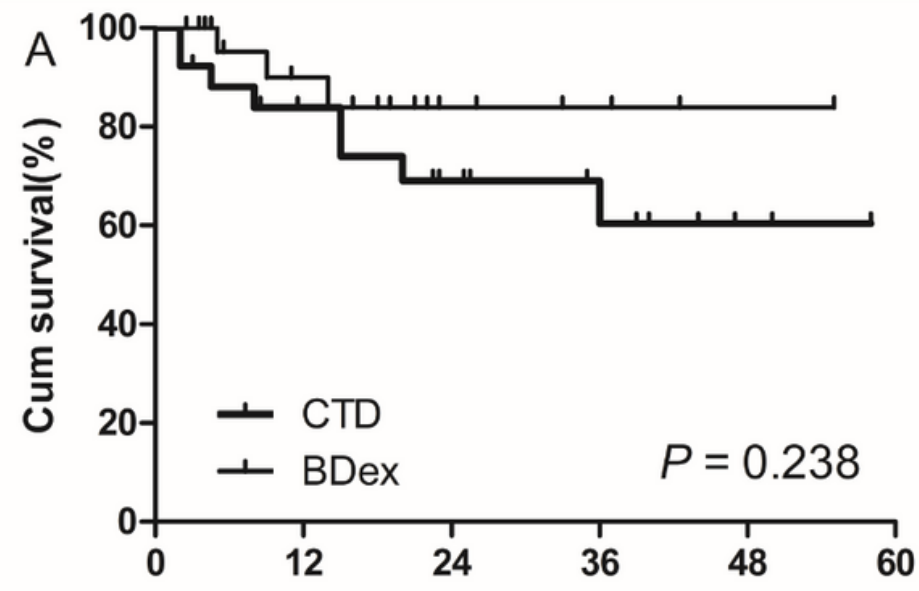

Patients at risk

CTD 26

BDex 26
Time (mo)

$\begin{array}{ccccc}19 & 12 & 8 & 3 & 1 \\ 17 & 7 & 4 & 2 & 1\end{array}$

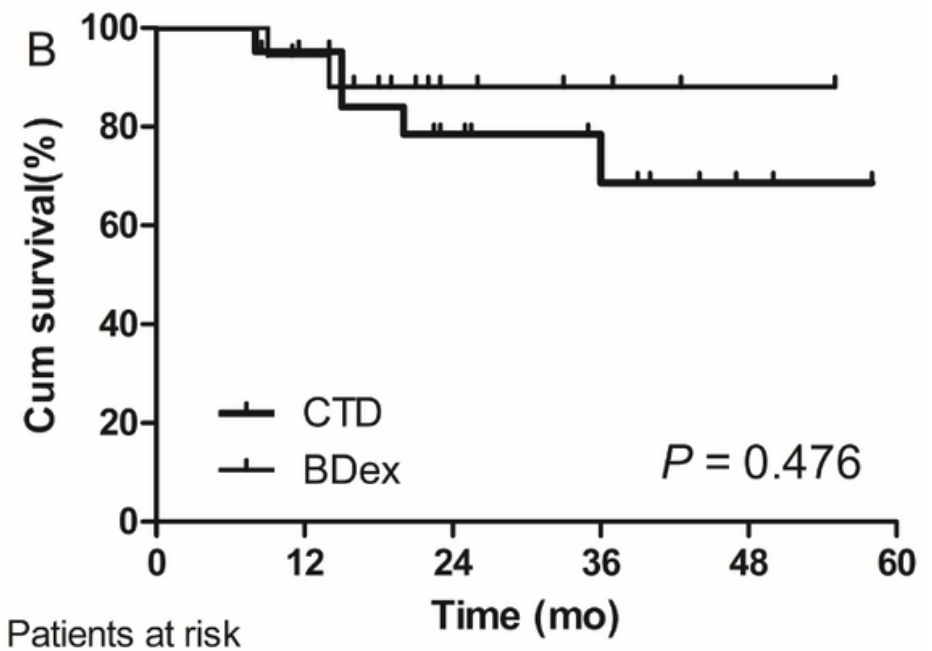

$\begin{array}{lllllll}\text { BDex } & 18 & 17 & 7 & 4 & 2 & 1\end{array}$

Figure 2

Overall survival of patients according to treatment type based on ITT analysis (A) and 6-month landmark analysis (B) after matching by Mayo stage 2012. 


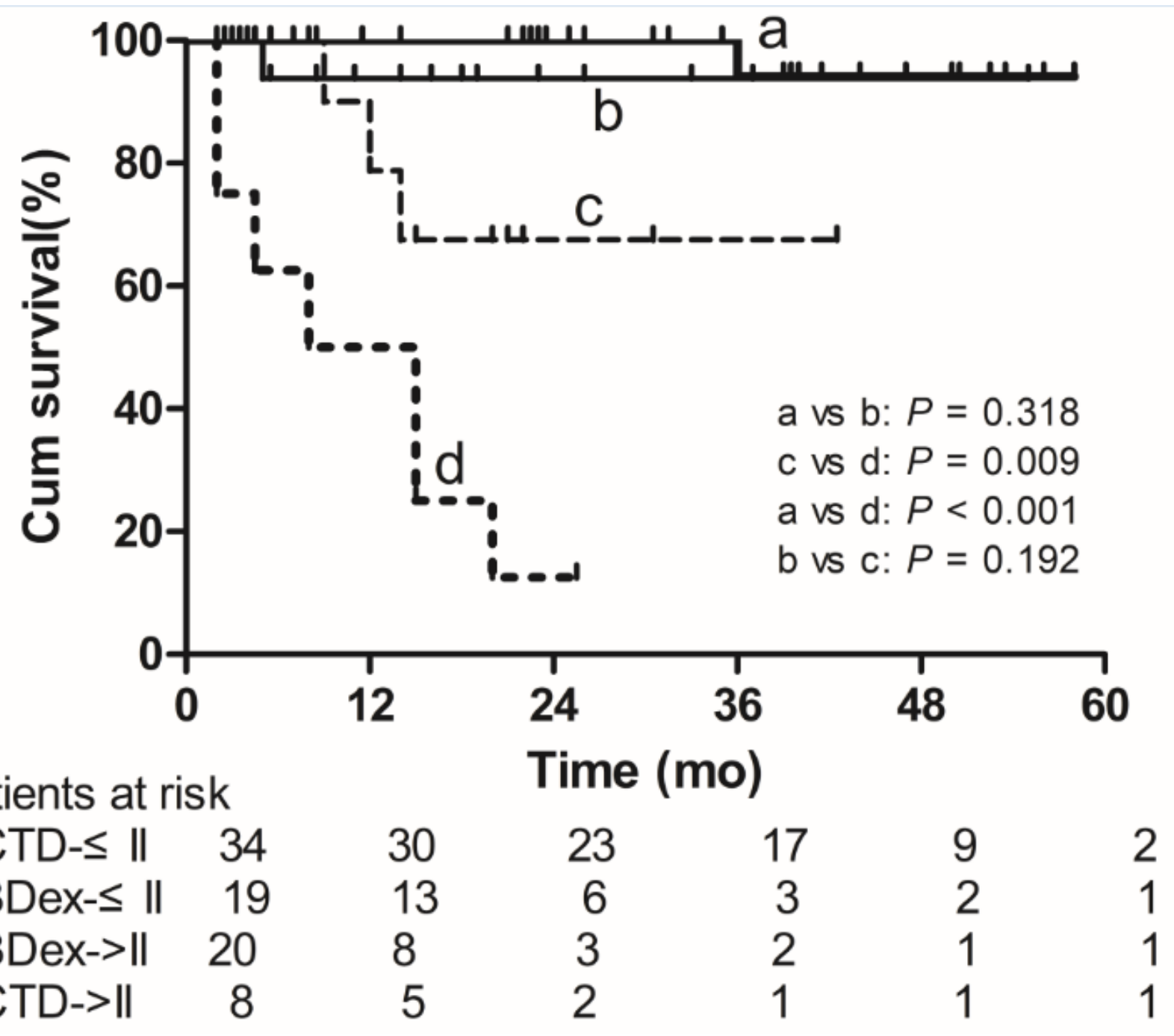

Figure 3

Overall survival of patients according to Mayo stage 2012 and treatment type. (a) Mayo stage $\leq$ II treated with CTD. (b) Mayo stage $\leq$ II treated with BDex. (c) Mayo stage $>$ II treated with BDex. (d) Mayo stage $>$ II treated with CTD. 